\title{
Planning and Control of Meso-scale Manipulation Tasks with Uncertainties
}

\author{
Peng Cheng David J. Cappelleri Bogdan Gavrea Vijay Kumar \\ GRASP Laboratory \\ University of Pennsylvania \\ Philadelphia, PA 19104 USA \\ \{chpeng, dcappell, gavrea, kumar\}@grasp.upenn.edu
}

\begin{abstract}
We develop a systematic approach to incorporating uncertainty into planning manipulation tasks with frictional contacts. We consider the canonical problem of assembling a peg into a hole at the meso scale using probes with minimal actuation but with visual feedback from an optical microscope. We consider three sources of uncertainty. Because of errors in sensing position and orientation of the parts to be assembled, we must consider uncertainty in the sensed configuration of the system. Second, there is uncertainty because of errors in actuation. Third, there are geometric and physical parameters characterizing the environment that are unknown. We discuss the synthesis of robust planning primitives using a single degreeof-freedom probe and the automated generation of plans for meso-scale manipulation. We show simulation and experimental results in support of our work.
\end{abstract}

\section{INTRODUCTION}

Manipulation and assembly tasks are typically characterized by many nominally rigid bodies coming into frictional contacts, possibly involving impacts. Manipulation tasks are difficult to model because uncertainties associated with friction and assembly tasks are particularly hard to analyze because of the interplay between process tolerance and geometric uncertainties due to manufacturing errors. Manipulation at the meso (hundred microns to millimeters) and micro (several microns to tens of microns) scale is even harder because of several reasons. It is difficult to measure forces at the micro-netwon level reliably using off-the-shelf force sensors and good forcefeedback control schemes have not proved successful. It is hard to manufacture general-purpose end effectors at this scale and it is even more difficult to grasp and manipulate parts at the micro and meso level than it is at the macro level. Finally, the lack of good models of the mechanics of contact interactions at this scale means that model-based approaches to planning and control are difficult.

The mechanics of pushing operations and sliding objects have been extensively studied in a quasi-static setting in [19, 22]. There is also extensive work addressing the analysis and simulation of mechanical systems with frictional contacts [3, 14, 5]. In particular, semi-implicit and instantaneous-time models for predicting motion and contact forces for quasistatic multi-rigid-body systems have recently been developed $[25,27]$. We build on these models and time-stepping algorithms discussed in these papers.

Modeling dry friction is a notoriously difficult problem area. Estimations of friction parameters for pushed objects to

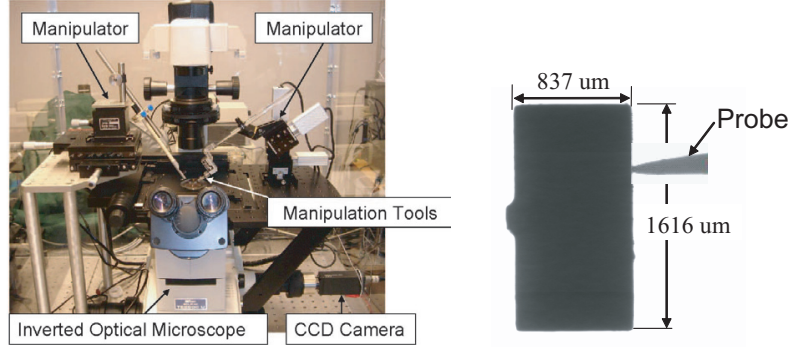

Fig. 1. Our experimental setup (left) and an image from the optical microscope showing the peg and a probe (right).
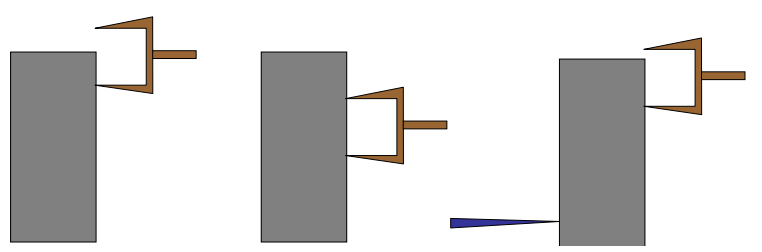

Fig. 2. Planar manipulation with a single degree-of-freedom, dual-tip probe and a passive single-tip probe. There are three sets of operations that can be performed.

improve the control of pushing have been investigated previously on larger objects and with different strategies than the ones presented here. In [17], test pushes on different objects with known support surfaces are used to estimate support surfaces experimentally. It leaves the open question of how the hypothesized support points for an unknown object should be chosen. Similarly in [28], a method for estimating the friction distribution of an object and the center of friction from pushing the object several times is presented. In both of these papers, a grid system of $N$ possible support points is applied to the base of the object being pushed. The respective algorithms determine the distribution of the normal force of the object at these support locations. Similarly, estimates of surface friction for meso-scale manipulation are experimentally determined in [7]. In our experiments the support surface is coated with a thin film of oil which circumvents the difficulties of modeling dry friction.

A good survey of motion planning under uncertainty is available in $[10,15]$. Pushing operations and the instantaneous motions of a sliding object during multiple contact pushing is examined and the manipulation primitive of stable rotational 
pushing is defined in [16]. In [2], the bounds of the possible motions of a pushed object are investigated. [23] presents a comparison between the dynamic and quasistatic motions of a push object. It is well-known that open-loop motion strategies, without the use of sensors, can be used to eliminate uncertainty and to orient polygonal parts [13, 11, 1]. In many cases, the problem of positioning and orienting a planar object with a random initial condition can be reduced to a planning problem which can be solved using a complete, polynomialtime algorithm.

In particular, the problem of finding motion primitives that rely on pushing and are robust to errors has received significant attention. A pushing control system with visual feedback for open-loop pushing is described in [24] as a way to mitigate the instability of pushing with point contacts. To remove the uncertainty associated with robot pushing tasks, [4] establishes stable orientation and positions by pushing objects with twopoint fingers. The problem of planning pushing paths using stable pushes with line contact is discussed in [18], and conditions on the pushing directions are derived that ensure that line sticking contact will always be maintained.

Sensorless orientation of parts is applied to micro-scale parts in [20]. At the micro scale, sticking effects due to Van der Walls forces and static electricity make the manipulator motions and part release more complicated $[12,6]$. Micromanipulators also have limited degrees of freedom when compared to manipulators at the macro-scale. These problems are addressed in [20] with a parallel-jaw gripper and squeeze and roll primitives to orient a randomly oriented polygonal part up to $180^{\circ}$ symmetry.

In this paper, we develop a formulation of the motion planning problem for manipulation with friction contacts incorporating uncertainty at three levels: (a) Errors in estimates of states (positions and orientations) of the manipulated object; (b) Errors in actuation or input; and (c) Errors in geometric and physical parameters characterizing the assembly task. We consider the canonical problem of assembling a planar, rectangular part into a planar, rectangular slot, but with a single degree-of-freedom probe with two rigid finger tips and a second passive probe (Fig. 2), and with visual feedback from an optical microscope (see Fig. 1). We address the automated generation of motion plans for this assembly task. We argue that it is appropriate to consider a quasi-static model of the manipulation task with Coulomb friction at the contact(s) between the probe and the object. The interaction between the manipulated part and the oil-coated surface is modeled using a viscous friction model. We explicitly model all three sources of uncertainty through experiments and show how motion primitives that are robust to these uncertainties can be identified. We describe a motion planner that can find motion plans composed of motion primitives for this task and illustrate the application through simulation and experiments.

\section{Modeling And Definitions}

The manipulation problem considered in this paper can be studied in the framework of motion planning for systems that are subject to both differential equations and uncertainties. In this section, we will briefly describe the framework for the general problem and a general planning methodology based on robust motions. The application of the general method in the manipulation problem is described in Section IV.

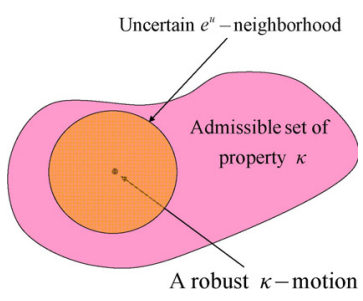

Fig. 3. Robust motion primitives

\section{A. Problem description}

Assume that the motion of the control system in the given environment is characterized by $\dot{x}=f(x, u, p)$, in which $x \in$ $X \subset \Re^{n}$ is the state, $u \in U \subset \Re^{m}$ is the input, and $p \in$ $P \subset \Re^{l}$ is the parameters for the system and environment. Given a control $\tilde{u}:\left[0, t_{\tilde{u}}\right] \rightarrow U$, a parameter history $\tilde{p}:$ $\left[0, t_{\tilde{p}}\right] \rightarrow P$, and a state $x_{0} \in X$ for some $t_{\tilde{u}}>0$ (varies with $\tilde{u})$, the trajectory (a.k.a. motion) under $\tilde{u}$ and $\tilde{p}$ from $x_{0}$ is $\tilde{x}\left(\tilde{u}, \tilde{p}, x_{0}, t\right)=x_{0}+\int_{0}^{t} f(\tilde{x}(\eta), \tilde{u}(\eta), \tilde{p}(\eta)) d \eta$.

We consider three bounded uncertainties stemming from sensing, control (actuation), and the environment.

1. Sensing uncertainty We assume that sensors can estimate the global state of the system with bounded error $s_{x}^{u}$. Let $x$ and $x^{s}$ respectively represent the actual and sensed states of the system. We have $x \in B_{s_{x}^{u}}\left(x^{s}\right)$, in which $B_{r}\left(x^{\prime}\right)=$ $\left\{x \mid\left\|x, x^{\prime}\right\| \leq r\right\}$ is the $r$-neighborhood of state $x$ with respect to a metric $\|\cdot, \cdot\|$ on $X$.

2. Control uncertainty We assume that actuators will realize the commanded control with a bounded error $c_{\tilde{u}}^{u}$. Let $\tilde{u}$ and $\tilde{u}^{i}$ respectively represent the actual and intended controls for the system. We have $\tilde{u} \in B_{c_{u}^{u}}\left(\tilde{u}^{i}\right)$.

3. Modeling uncertainty $\mathrm{We}$ assume that the geometry and the physics of the underlying model are parameterized by $\tilde{p}$ with bounded error $e_{\tilde{p}}^{u}$. Let $\tilde{p}$ and $\tilde{p}^{n}$ respectively represent the actual and nominal parameter history. We have $\tilde{p} \in B_{e_{\tilde{p}}^{u}}\left(\tilde{p}^{n}\right)$.

Given a sensed initial state $x_{\text {init }}$ and a goal set $X_{\text {goal }}^{p}=$ $B_{\tau}\left(x_{\text {goal }}\right)$ for a specified $\tau$ and $x_{\text {goal }}$, the objective is to compute a control $\tilde{u}$ (that may depend on feedback information) which will drive the system from $x_{\text {init }}$ to $X_{\text {goal }}$ under uncertainties.

\section{B. Planning with robust motion primitive}

To solve the above problem is quite difficult. Because complete algorithms are difficult to find except for the simplest of problems, we pursue the synthesis of plans that are obtained by composing robust motion primitives. Robust motion primitives are used to define controls whose resulting trajectories will preserve a specified property of interest in the presence of uncertainties. We model a property of interest by a characteristic function, $\kappa$, which maps a trajectory into 0 or 1 . If $\kappa(\tilde{x})=1$, then we say that the trajectory $\tilde{x}$ satisfies the given property and is called a $\kappa$-motion. The admissible set for a property $\kappa$ (see Fig. 3) is $\mathcal{A}_{\kappa}=\{\tilde{x} \mid \kappa(\tilde{x})=$ $1\}$. If the system has uncertainty bound $e^{u}=\left(s_{x}^{u}, c_{\tilde{u}}^{u}, e_{\tilde{p}}^{u}\right)$, the uncertainty neighborhood of trajectory $\tilde{x}=\left(x_{0}, \tilde{u}, \tilde{p}\right)$ is $\left\{\tilde{x}^{\prime} \mid\left\|x_{0}^{\prime}, x_{0}\right\| \leq s_{x}^{u},\left\|\tilde{u}^{\prime}, \tilde{u}\right\| \leq c_{\tilde{u}}^{u},\left\|\tilde{p}^{\prime}, \tilde{p}\right\| \leq e_{\tilde{p}}^{u}\right\}$. A $\kappa-$ motion is a robust motion primitive only if its uncertainty neighborhood is contained within the admissible set.

We can now consider the composition of robust motion primitives. Let $\kappa_{1}$ and $\kappa_{2}$ be two properties. If there exists 
a robust $\kappa_{1}$-motion and a robust $\kappa_{2}$-motion such that the $\kappa_{1}$-motion can be reliably appended to the $\kappa_{2}$-motion under uncertainties, then we say that it is possible to sequentially compose the motion primitives.

Thus our approach to planning will involve the construction of a set of robust motion primitives followed by their sequential composition. At this point, a graph search based motion planning algorithm in [15] can be used to synthesize the complete motion plan. It is worth mentioning that such algorithms are not complete because they restrict the search space from the original control space to a smaller one consisting only of robust motion primitives.

In the next section we will describe our experimental testbed and the specifics of the manipulation task before developing models of the manipulation task and robust motion primitives for the task.

\section{ThE EXPERIMENTAL TESTBED}

The mico-manipulation system (Fig. 1 left) consists of an inverted optical microscope and CCD camera (for sensing the configuration), 4 axis micro-manipulator, controller, $5 \mu \mathrm{m}$ tip tungsten probes, and control computer. There is a $4 \mathrm{X}$ objective on the microscope along with a $0.6 \mathrm{X}$ optical coupler producing a field of view (FOV) of $3.37 \mathrm{~mm} \mathrm{x} 2.52 \mathrm{~mm}$. The CCD camera records the images in the FOV and sends them to the control computer at $30 \mathrm{~Hz}$ (lower frequency with image processing). The micro-manipulator with controller has a minimum incremental motion of $0.1 \mu \mathrm{m}$ along four axes, with a maximum travel of $20 \mathrm{~mm}$ and with speeds ranging from $1.6 \mu \mathrm{m} / \mathrm{sec}$ to $1.7 \mathrm{~mm} / \mathrm{sec}$. We consider two types of probes, a passive Single-Tip Probe (STP) and an active DualTip Probe (DTP). The STP is passive and although it can be positioned, its motion is not controlled during manipulation. The DTP is actuated along one direction (the $x$-axis) and can be used either for single or two point contact (see Fig. 2).

The control of the DTP is fully characterized by $u=$ $\left(d_{2}, v_{p}, p^{t}\right)$ (see Fig. 3), denoting a push in $x$ direction with relative distance $d_{2}$ with duration $p^{t}$ and constant speed $v_{p}$. In the experiments in this paper, we choose from one of three discrete values of speeds: $v_{p}=140.0,75.0$ or $7.4 \mu \mathrm{m} / \mathrm{sec}$. The other two inputs are continuous.

As mentioned before, there are three sources of uncertainty. The sensing uncertainty arises because of the limitation on the magnification and resolution of the camera. Because with our objective, each pixel subtends only $5.26 \mu \mathrm{m}$, our errors in positions are approximately $\pm 5 \mu \mathrm{m}$ and the error in estimating the orientation of our $1616 \mu \mathrm{m} \times 837 \mu \mathrm{m}$ part is \pm 0.3 degrees. The control uncertainty exists only in the probe position. The errors in probe position relative to the part are also of the order of $\pm 5 \mu \mathrm{m}$. Errors in geometric parameters stem from manufacturing imperfections. The part is not a perfect rectangle as shown in Fig. 1. The tips in the DTP are of different length, in which one tip is longer than the other, reflected in the angle $\beta$ in Fig. 4 (right). However, we assume the exact dimensions are known. The principal source of modeling error stems from surface friction and the coefficient of friction between the probe(s) and the part. We will discuss the dynamic model and the parameters governing this model in greater detail in the next section.

\section{Motion Planning With UnCERTAinty}

\section{A. System dynamics}

We use a quasi-static model for the system (inertial forces are of the order of nano-newtons for the accelerations involved, while the frictional forces are on the order of micro-newtons). We assume the support plane to be uniform, and all pushing motions of the probes to be parallel to this plane. The most important assumption is about the support friction. Because we coat the support surface with oil (Extra Heavy Mineral Oil, LSA, Inc.), it is reasonable to assume viscous damping at the interface. Based on experimental data we chose the model $f=E v$ in which $v=\left[v_{x}, v_{y}, v_{\theta}\right]^{T}$ is the velocity of the part (peg) configuration $x, y, \theta ; f$ is the corresponding vector of forces and moments; and $E$ is the damping diagonal matrix with diagonal elements $e_{x}, e_{y}=e_{x}$, and $e_{\theta}$. The coefficient of friction between the probe and the part is $\mu$. These parameters were computed by parameter fitting with experimental results (see Section V-A). Finally, we assume the only contacts that occur are between the probe and the part. Although we consider the assembly task as our goal, we only consider the problem of guiding the part into the designated slot without any collisions with the environment.

From quasi-static analysis, we have $E v=$ $\sum_{i}\left(w_{n}^{i} \lambda_{n}^{i}+w_{t}^{i} \lambda_{t}^{i}\right)$, where $w$ denotes the wrench vector and $\lambda$ the magnitude of the contact force, with subscripts $n$ and $t$ indicating normal and tangential directions, and the superscript $i$ denoting the $i^{t h}$ contact. Because of space constraints, we do not write the entire model which includes complementarity constraints for sticking, sliding and separation, but instead refer the reader to [21, 27].

We note that the existence of a trajectory for the rigid-body quasi-static model described above may be guaranteed under the assumption that the generalized friction cone is pointed (by pointed cone, we mean a cone that doesn't contain any proper linear subspace). The proof of this result follows the lines of [26] but is omitted because of space constraints. Therefore, for the one-point contact case in Fig. 2 (left), existence is immediately obtained from the linear independence of the normal and tangential wrenches. When the probe pushes the same side of the part with a two-point contact (Fig. 2 (center)), it is also easy to see that the friction cone is again pointed, and thus a solution will always exist. The remaining twopoint contact case corresponds to the two point contact in Fig. 2 (right), for which it can be shown that the pointedness of the friction cone holds if the distance between the points of contact is large enough. This motion, which can be used to rotate the part is discussed later in the next subsection. Finally, if we derive robust motion primitives that guarantee sticking is maintained at one or two contact points, we automatically obtain uniqueness of the trajectories by using traditional arguments with the underlying differential algebraic equation. We note that the uniqueness of contact forces does not hold in general, even though part's trajectory is guaranteed to be unique.

\section{B. Properties of motions and admissible sets}

There are many properties of interest for pushing primitives for our meso-scale manipulation task, e.g., inputs that guarantee motions with one (or two) sticking contacts or input that guarantee desired clockwise (or counter clockwise 

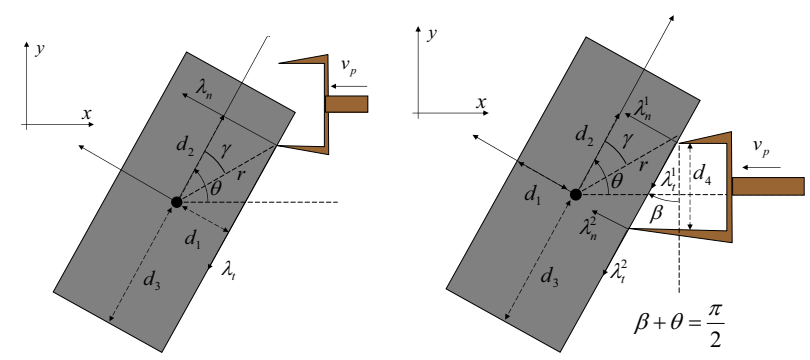

Fig. 4. Pushing with one-point (left) and two-point contact (right). In the right picture, the DTP is shown with the exaggerated misalignment between its two tips for better visualization.

rotation) of the part. In the following, we will specially discuss three types of properties for which robust motions can be systematically constructed. The first property is to maintain the one-point sticking contact with counter clockwise (or clockwise) rotation. The second property is to maintain the two-point sticking contact for the DTP. The third property is that the orientation of the final state of the motion is close to 0 or $\pi$ radians (because the slot is horizontally oriented). Sufficient conditions for motion primitives that guarantee each of these properties are presented below.

1) One-point sticking contact with counter clockwise rotation: We only consider the case in which $\theta \in(0, \pi)$ and the probe pushes on the long edge of the part (see Fig. 4 left). However, other cases, such as pushing on the short edge or the right side of the part, can be analyzed similarly.

The following provides the conditions for a static point:

$$
\begin{aligned}
\left|\frac{\lambda_{n}}{\lambda_{t}}\right| & =\left|\frac{d_{1} d_{2} e_{x} \cos \theta+\left(e_{\theta}+d_{1}^{2} e_{x}\right) \sin \theta}{\left(e_{\theta}+d_{2}^{2} e_{x}\right) \cos \theta+d_{1} d_{2} e_{x} \sin \theta}\right|>\frac{1}{\mu} \\
\lambda_{n} & =-\frac{e_{x} v_{p}\left(d_{1} d_{2} e_{x} \cos \theta+\left(e_{\theta}+d_{1}^{2} e_{x}\right) \sin \theta\right)}{f_{d}(x, u, E)}>0 \\
v_{\theta} & =\frac{e_{x} v_{p}\left(d_{1} \cos \theta-d_{2} \sin \theta\right)}{f_{d}(x, u, E)}>0
\end{aligned}
$$

in which $f_{d}(x, u, E)=e_{\theta}+\left(d_{2}^{2}+d_{1}^{2}\right) e_{x}>0$ and $v_{p}<0$.

From (1), we can infer the property of the whole motion just from its initial point, which is stated in the following lemma:

Lemma 1: If the part starts a counter clockwise rotation with sticking contact at the initial point with orientation $\theta \in$ $(0, \pi)$ (satisfying (1)) as shown in Fig. 4 (left), then the part will keep counter clockwise rotation with sticking contact until its orientation $\theta$ reaches

$$
\pi-\max \left\{\tan ^{-1} \frac{d_{1}}{d_{2}}, \tan ^{-1} \frac{d_{1} d_{2} e_{x}}{e_{\theta}+d_{1}^{2} e_{x}}\right\} .
$$

Proof: The derivatives of $\frac{\lambda_{n}}{\lambda_{t}}$ and $v_{\theta}$ with respect to $\theta$ are as follow:

$$
\begin{aligned}
\frac{\partial\left(\lambda_{n} / \lambda_{t}\right)}{\partial \theta} & =\frac{e_{\theta}\left(e_{\theta}+\left(d_{1}^{2}+d_{2}^{2}\right) e_{x}\right)}{\left(\left(e_{\theta}+d_{2}^{2} e_{x}\right) \cos \theta+d_{1} d_{2} e_{x} \sin \theta\right)^{2}} . \\
\frac{\partial v_{\theta}}{\partial \theta} & =-\frac{e_{x} v_{p}\left(d_{2} \cos \theta+d_{1} \sin \theta\right)}{e_{\theta}+\left(d_{1}^{2}+d_{2}^{2}\right) e_{x}} .
\end{aligned}
$$

It can be observed that both derivatives are strictly positive before $\theta$ reaches (2). Therefore, if the part rotates counter clockwise $\left(v_{\theta}>0\right)$ in the sticking mode $\left(\left|\frac{\lambda_{n}}{\lambda_{t}}\right|>\frac{1}{\mu}\right)$ at the initial point, then the part will keep staying in the sticking

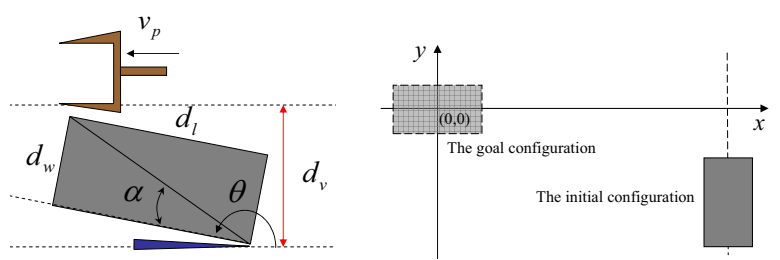

Fig. 5. The robust rotational motion and planning problem setup

mode because $\frac{\lambda_{n}}{\lambda_{t}}$ will keep increasing and $v_{\theta}$ will keep strictly positive as $\theta$ increases.

2) The two-point sticking contact: We only describe the case in which $\theta \in(0, \pi)$ and the DTP pushes on the long edge of the part and the contact is sticking (see Fig. 4 right).

The following equations ensure that two point contact will be sticking at a static point:

$$
\begin{aligned}
\left|\frac{\lambda_{n}}{\lambda_{t}}\right| & =|\tan \theta|=|1 / \tan \beta|>\frac{1}{\mu} \\
\lambda_{n}^{1} & =\frac{e_{x} v_{p} \cos \beta\left(d_{1} \sin \beta+d_{2} \cos \beta-d_{4}\right)}{d_{4}}>0 \\
\lambda_{n}^{2} & =-\frac{e_{x} v_{p} \cos \beta\left(d_{1} \sin \beta+d_{2} \cos \beta\right)}{d_{4}}>0
\end{aligned}
$$

The following lemma shows whether the whole motion has a two-point sticking contact can be determined from the initial point.

Lemma 2: If the part starts with two-point sticking contact as shown in Fig. 4 (right), then the pushing will stay in the two-point sticking contact mode.

Proof: It is because (5) depends on the orientation and the orientation is invariant when the initial point has the two-point sticking contact.

3) The orientation of the final state is close to 0 or $\pi$ radians: This property will be achieved in a motion by pushing the part with the active DTP with a separation

$$
d_{v} \geq d_{w}+2 s_{x}^{u}+2 c_{\tilde{u}}^{u} .
$$

to the passive STP to guarantee the intended rotation under sensing and control uncertainties (see Fig. 5 left). Such pushing will ensure that the final orientation will be in $\theta_{t^{-}}$ neighborhood of $\pi$, in which

$$
\theta_{t}=\sin ^{-1} \frac{d_{w}+2 s_{x}^{u}+2 c_{\tilde{u}}^{u}}{\sqrt{d_{w}^{2}+d_{l}^{2}}}-\alpha .
$$

Remark: In order to guarantee existence, the pointed cone assumption requires $d_{v} \geq d_{w} \tan \sigma$ where $\sigma=\tan ^{-1} \mu$ is the angle of the friction cone. This is clearly satisfied by (6). However, for this motion we cannot guarantee uniqueness of the resulting trajectory. In this case, the property of interest (the desired change in orientation) does not depend on the specifics of the trajectory and thus the lack of a guarantee on uniqueness is not a problem.

\section{Computing robust motions from the admissible sets}

We use a simple sampling-based algorithm to find a robust motion with respect to a given property at a given state. We incrementally decrease the sampling dispersion along each dimension of the input space until the dispersion reaches the respective control uncertainty bounds. Initially, the sampling 
dispersion in each dimension of the input space is chosen to be the half of the maximal distance. In each iteration, the sample points in each dimension with respect to its current sampling dispersion are combined to generate all possible inputs. Each input is tested for membership in the admissible set under the nominal state and parameters. If no input is in the admissible set, then the sampling dispersion is decreased by half and the algorithm goes into the next iteration. If an input is in the admissible set under the nominal state and parameters, then this input is tested to see whether it is still in the admissible set under the maximal uncertainties in sensing, control, and parameters. If yes, then the motion from this input is returned as a robust motion. If no robust motion is found when the algorithm stops, then there exists no robust motion with respect to the given property under such uncertainties.

\section{Comparison of robust motions}

As we show in Section IV-B, there might exist many types of robust motions with respect to different properties. In this section, we will provide a measure, the Lipschitz constant of the motion equation, to compare robustness of these motions with respect to uncertainties.

The Lipschitz constants have been used before to provide an upper bound on the variation of the trajectory with respect to changes in the state, control, and parameters [8, 9]. The magnitude of Lipschitz constants characterizes the worst case trajectory variation of the system under uncertainties. If the Lipschitz constant is smaller, then the upper bound on the trajectory variation with respect to uncertainties is smaller, i.e., the corresponding motion will be more robust.

We compute the Lipschitz constants with respect to the fixed initial part configuration $(x, y, \theta), d_{2}, E$ (damping matrix), and $\mu$ (friction coefficient) for motion equations of the part under the push of the STP and DTP with the same probe initial position (the top tip for the DTP and the tip of the STP have the same position), constant fixed velocity, and time duration. It is shown that those constants for the STP are greater, and therefore the DTP has less uncertainty than the STP with respect to this measure. This result is supported by the experimental results in Section V.

\section{E. Planning with robust motion primitives}

The assembly task considered in the paper has the initial configuration with orientation $\frac{\pi}{2}$ and a goal configuration of $(0,0,0)$ with position tolerance of $\epsilon_{p}=76 \mu \mathrm{m}$ and orientation tolerance $\epsilon_{\theta}=5^{\circ}$ (see Fig. 5 right). Note that we currently ignore obstacles in the environment. For such a task, our planning algorithm relies on composing the simple robust motions defined above. We first construct the following three higher level robust motion primitives using these simple ones. 1. Robust translation in the $x$ direction

This robust translation is achieved by using DTP to push the part in the $x$ direction while maintaining two-point sticking contact. However, because the two tips of a DTP may not be aligned (see $\beta$ in Fig. 4 right) or sensing errors exist, two point contact might not be established, or can only be established after the one-point contact is established first at either the top or the bottom tip. To increase robustness, we define a to-two-contact property, denoted as $t^{2}$, by a sequential composition of a one-point sticking contact motion with a counter clockwise rotation followed by a two-point sticking

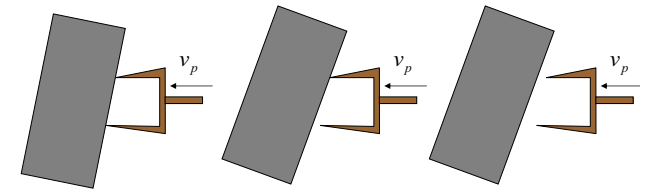

Fig. 6. The $t^{2}$ motion starting from the right figure to the left.

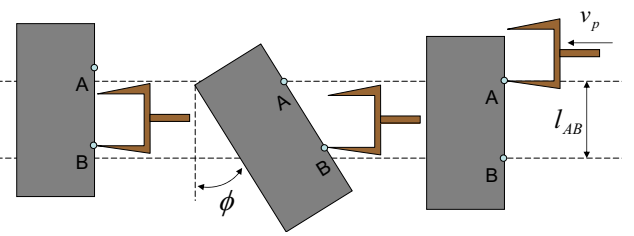

Fig. 7. Vertical translational motion starting from the right figure to the left.

contact motion (see Fig. 6). Lemmas 1 and 2 respectively provide conditions for one-point and two-point sticking contact motions. The following lemma will ensure that two sticking motions can be combined to ensure a $t^{2}$ motion.

Lemma 3: Assume that the top tip first establishes the contact. When the misalignment parameter, $\beta$, of the DTP satisfies

$$
|\tan \beta|<\min \left\{\mu, \frac{d_{2}}{d_{1}}, \frac{d_{1} d_{2} e_{x}}{e_{\theta}+d_{2}^{2} e_{x}}\right\} ; \beta+\theta<\frac{\pi}{2},
$$

the counter clockwise rotation with one-point sticking contact can be followed by a two-point sticking motion.

Proof: The first inequality in (8) ensures that twopoint sticking contact is admissible and can be established before the one-point sticking contact motion stops. The second inequality ensures that a counter clockwise rotation with onepoint sticking contact will precede the two-point sticking contact motion.

\section{Robust translation in the $y$ direction}

This translation is achieved by composing a robust motion with one point sticking contact and intended rotation followed by a robust $t^{2}$ motion (see Fig. 7). The amount of the net vertical translation is $l_{A B}(1-\cos \phi)$ under nominal conditions (no uncertainty).

\section{Robust rotation}

This motion is achieved with the pushing described in Section IV-B.3.

Planning algorithm: With the above three higher level robust motion primitives, the planning algorithm consists of the following steps:

Step 1: Move in the $y$ direction by pushing along the long edge of the part such that $y \in\left[-\frac{\epsilon_{p}}{2}, \frac{\epsilon_{p}}{2}\right]$. We use a sequence of $y$-direction motions in Fig. 7, guaranteeing that the net $y$ translation of $l_{A B}(1-\cos \phi)$ in Fig. 7 will have the following error bound $d_{v}^{u}=\max \left\{d_{1}^{e}, d_{2}^{e}\right\}$, in which $d_{1}^{e}=\left|l_{A B}(1-\cos \phi)-\left(l_{A B}-2 s_{p}^{u}-2 c_{p}^{u}\right)\left(1-\cos \left(\phi-2 s_{\theta}^{u}\right)\right)\right|$, $d_{2}^{e}=\left|l_{A B}(1-\cos \phi)-\left(l_{A B}+2 s_{p}^{u}+2 c_{p}^{u}\right)\left(1-\cos \left(\phi+2 s_{\theta}^{u}\right)\right)\right|, s_{p}^{u}$ and $c_{p}^{u}$ are respectively the sensing and control error bounds in the position, and $s_{\theta}^{u}$ is the sensing error bound in the orientation. To ensure that $y \in\left[-\frac{\epsilon_{p}}{2}, \frac{\epsilon_{p}}{2}\right]$ can be achieved using the vertical primitive under sensing and control uncertainties, the following conditions on the uncertainty bounds must be satisfied: $s_{p}^{u}+d_{v}^{u} \leq \frac{\epsilon_{p}}{2}, \phi>2 s_{\theta}^{u}, l_{A B}>2 s_{p}^{u}+2 c_{p}^{u}$.

Step 2: Rotate to $\theta=\pi$. As shown in (7) and Fig. 5 (left), the distance of the orientation of the part to the horizontal line 
TABLE I

Y-Translation Primitive: Net Displacement of the Part

\begin{tabular}{|c|c|c|c|}
\hline Test No. & $X(\mu \mathrm{m})$ & $Y(\mu \mathrm{m})$ & $\theta$ \\
\hline 1 & 1996 & 19 & $0.6^{\circ}$ \\
\hline 2 & 1975 & 18 & $0.5^{\circ}$ \\
\hline 3 & 1559 & 20 & $1.1^{\circ}$ \\
\hline Average & 1843 & 19 & $0.7^{\circ}$ \\
\hline Simulation & 1443 & 11 & $0.0^{\circ}$ \\
\hline Theory & $N A$ & 11 & $0.0^{\circ}$ \\
\hline
\end{tabular}

will be bounded. To ensure that the final $t^{2}$ pushing can be robustly applied, we require that uncertainty bounds satisfy: $\theta_{t}=\sin ^{-1} \frac{d_{w}+2 s_{p}^{u}+2 c_{p}^{u}}{\sqrt{d_{w}^{2}+d_{l}^{2}}}-\alpha<\theta_{t^{2}}^{\max }$, in which $\theta_{t^{2}}^{\max }$ is the maximal orientation of the part allowing a robust $t^{2}$ pushing and can be computed using the algorithm in Section IV-D.

Step 3: If necessary, move in the $y$ direction by pushing along the short edge of the part such that $y \in\left[-\frac{\epsilon_{p}}{2}, \frac{\epsilon_{p}}{2}\right]$.

Step 4: Translate the part in $x$ direction to the goal $(0,0,0)$. With the robust $t^{2}$ motion primitives, the final configuration of part will be $x \in\left[p^{x}+r \cos (\gamma+\beta)-c_{p}^{u}, p^{x}+r \cos (\gamma+\right.$ $\left.\beta)+c_{p}^{u}\right], y \in\left[p_{y}-r \sin (\gamma+\beta)-c_{p}^{u}, p_{y}-r \sin (\gamma+\beta)+c_{p}^{u}\right]$, and $\theta=\beta$ in which $p^{x}, p^{y}$ is the position of the top tip of the DTP, $d_{2}, r$ and $\gamma$ are as shown in Fig. 4 (right). These equations also impose restrictions on the uncertainty bounds to ensure the intended tolerance: $r_{\max }\left(\cos \left(\gamma_{\max }-\beta_{\max }\right)-\right.$ $\left.\cos \gamma_{\max }\right)+2 c_{p}^{u}<\epsilon_{p}$ and $r_{\max } \sin \beta_{\max }+2 c_{p}^{u}<\epsilon_{p}, \beta_{\max }<$ $\epsilon_{\theta}$, in which $\gamma_{\max }=\tan ^{-1} \frac{d_{1}}{d_{3}}, r_{\max }=\sqrt{d_{1}^{2}+d_{3}^{2}}$, and $\beta_{\max }$ is the maximal magnitude for $\beta$ (see Fig. 4 right).

\section{SimUlation AND EXPERIMENTAL RESUlTS}

We did a series of experiments to estimate the parameters (including the damping matrix and friction coefficient) for the system and to compare robust and non-robust motions using both the DTP and STP. In the next two subsections, we show representative results for the system identification and for the different motion primitives. In Section V-D, we used the designed planner to compute probe controls to complete a given task in both the simulation and experiment.

\section{A. Estimating of system parameters}

The parameter fitting was done with the experimental data obtained using the STP. Figure 8 shows experimental trajectories versus predicted trajectories for one trial that was used in the parameter estimation (top) and one trial that was not (bottom). To estimate the parameters, a root-mean-square metric is used. The optimization algorithm is derived from the Nelder-Mead method. The diagonal elements of damping matrix $E$ are estimated to be $e_{x}=e_{y}=160.89 \mathrm{~N} \cdot \mathrm{sec} . / \mathrm{m}$ and $e_{\theta}=60.64 \mathrm{~N} \cdot \mathrm{m} \cdot \mathrm{sec}$. The coefficient of friction between the part and the probe is estimated to be $\mu=0.3 \sim 0.36$. These figures show $30-40 \mu \mathrm{m}$ position errors across a translation of about $600 \mu \mathrm{m}$ and about $3^{\circ}$ orientation errors for a $45^{\circ}$ rotation.

\section{B. Comparison between robust and non-robust motions}

Trajectories from robust motion primitives show less variation (and are therefore more predictable) than trajectories from other motion primitives. Figure 9 shows the experiment setup (top) and experimental trajectory plots for comparison of the robust and non-robust motions using the DTP and STP. Tests 1
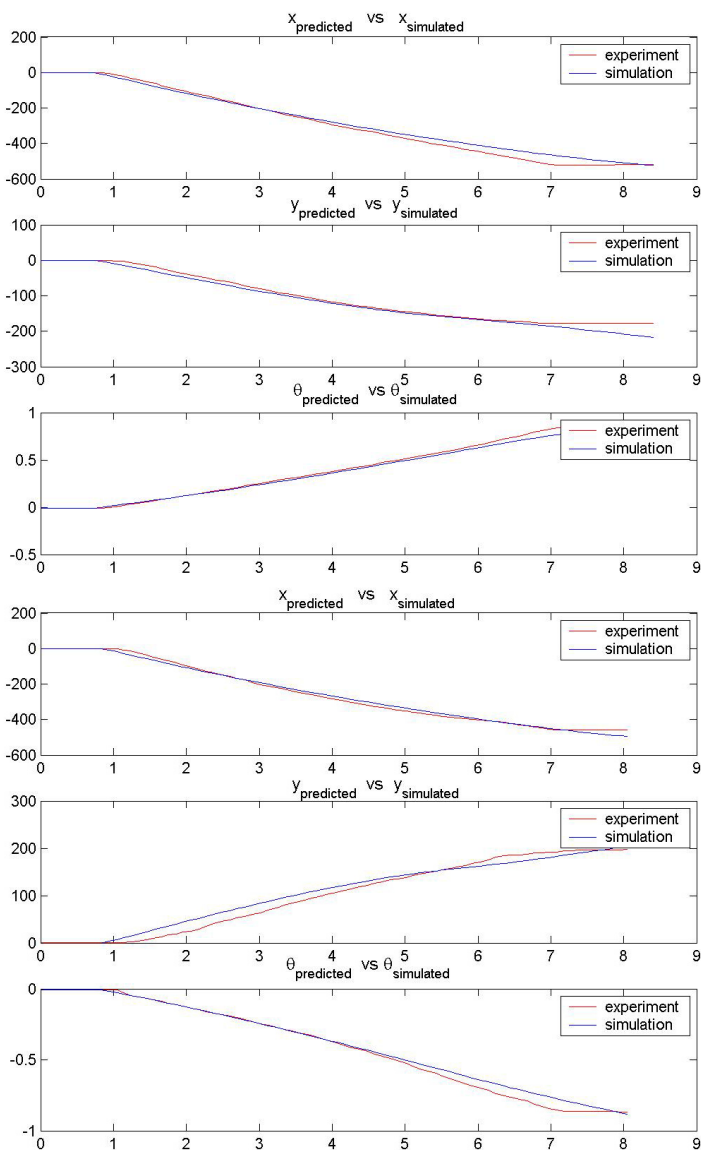

Fig. 8. System identification: The top three plots show a representative trial used for system identification and the bottom three plots show a representative trial used to verify the model.

and 2 are for robust and non-robust $t^{2}$ motions with the DTP. Test 1 was verified to satisfy the robust $t^{2}$ motion conditions in Sections IV-B.1 and IV-B.2. The experiments showed that the two-point contact is well maintained because the orientation $\theta$ is almost constant after the two point contact is established. Test 2 did not satisfy the two-point sticking contact conditions, and therefore the two point contact was broken once it was established. We also observed that Test 1 has maximal trajectory differences of $20 \mu \mathrm{m}$ in $x, 15 \mu \mathrm{m}$ in $y$, and 0.023 radians in $\theta$, which are smaller than the corresponding numbers for Test 2 (maximal trajectory differences at $15 \mu \mathrm{m}$ in $x, 25 \mu \mathrm{m}$ in $y$, and 0.1 radians in $\theta$ ).

\section{Comparison between the DTP and STP}

Trajectories using the DTP show less variation than those obtained from the STP. Tests 1 and 3 in Fig. 9 are results from robust motion primitives for the DTP and STP respectively. The top tip of the DTP had the same $y$ position as the STP. Trajectories from Test 1 have less variation than those from Test 3, whose maximal trajectory differences are $75 \mu \mathrm{m}$ in $x$, $75 \mu \mathrm{m}$ in $y$, and 0.2 radians in $\theta$.

\section{Planning in both the simulation and experiment}

Table I shows the comparison between theoretical, simulated and experimental results for robust translation in the $y$ direction, for the motion primitive described in Section IV-E. Tables II and III compare the experimental and simulated results 

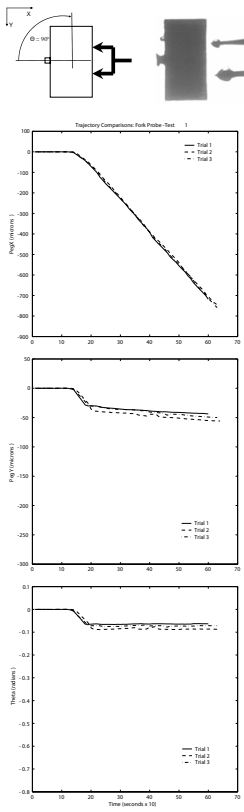

Test 1 (DTP) robust
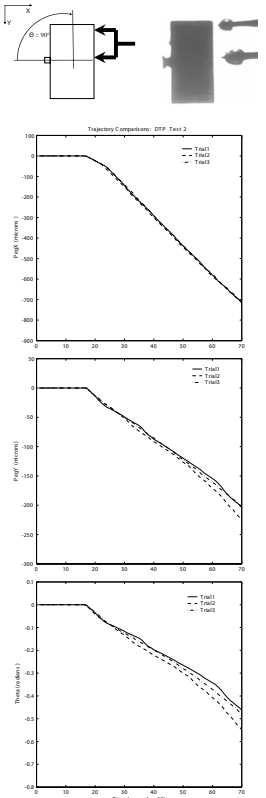

Test 2 (DTP) not robust
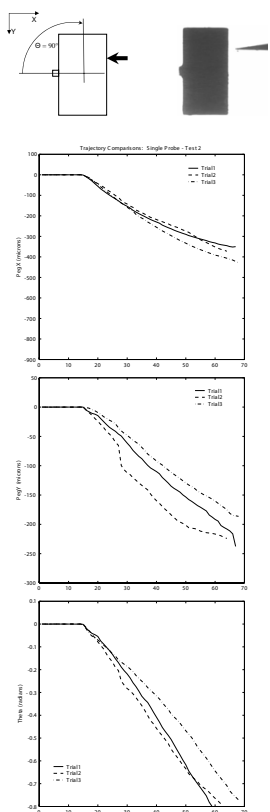

Test 3 (STP) robust

Fig. 9. Experimental results for robust and non-robust motions with the DTP and the STP.

TABLE II

Rotational Motion: Net Displacement of the Part

\begin{tabular}{|c|c|c|c|}
\hline Test No. & $X(\mu \mathrm{m})$ & $Y(\mu \mathrm{m})$ & $\theta$ \\
\hline 1 & 381 & 34 & $88^{\circ}$ \\
\hline 2 & 434 & 32 & $90^{\circ}$ \\
\hline 3 & 370 & 14 & $90^{\circ}$ \\
\hline Average & 382 & 27 & $89^{\circ}$ \\
\hline Simulation & 295 & 0.05 & $90^{\circ}$ \\
\hline
\end{tabular}

for executing robust rotational motion and robust translation in the $x$ direction motion, respectively. At least 3 experimental tests were done for each motion type and the average values of the tests are shown in the tables. For the robust $y$ translation tests, the initial robust one-point sticking contact is maintained until a desired part rotation angle, $\phi$, is achieved. This is then followed by a robust $t^{2}$ push to restore the part to the upright position. Simulation and theoretical results match very well for the $\phi$ tested. Experiments show a somewhat higher (7$9 \mu \mathrm{m})$ net displacement than the predicted $y$ translation, but it is likely due to measurement errors - errors in estimating position are $\pm 5 \mu \mathrm{m}$. We did not observe sliding in the pushing from the image analysis.

In the robust rotational motion experiments, the separation

TABLE III

X-Translation Primitive: Net Displacement of the Part

\begin{tabular}{|c|c|c|c|}
\hline Test No. & $X(\mu \mathrm{m})$ & $Y(\mu \mathrm{m})$ & $\theta$ \\
\hline 1 & 954 & 5 & $0.1^{\circ}$ \\
\hline 2 & 944 & 11 & $0.7^{\circ}$ \\
\hline 3 & 965 & 11 & $0.7^{\circ}$ \\
\hline 4 & 959 & 5 & $0.5^{\circ}$ \\
\hline 5 & 954 & 0 & $0.0^{\circ}$ \\
\hline Average & 955 & 6 & $0.4^{\circ}$ \\
\hline Simulation & 949 & 0.2 & $0.0^{\circ}$ \\
\hline
\end{tabular}

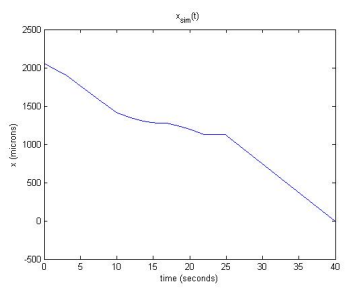

Simulation: $x$

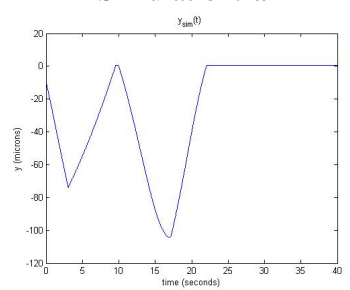

Simulation: $y$

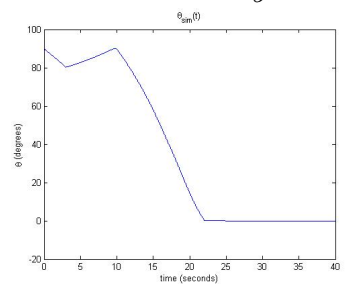

Simulation: $\theta$

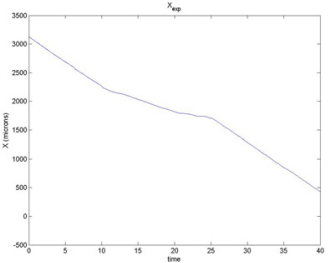

Experiment: $x$

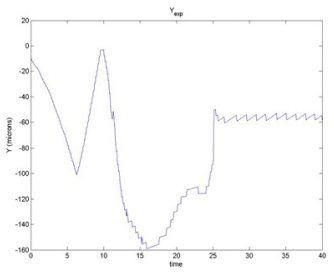

Experiment: $y$

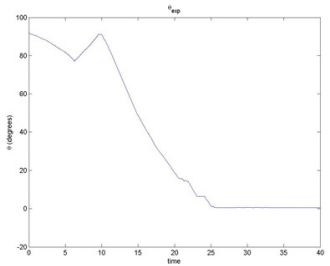

Experiment: $\theta$
Fig. 10. Simulation (left) and experimental (right) results for a planning task

distance is determined from (6) and the two probe tips are centered about the center of the part that has orientation $\pi / 2$. The STP probe is to the left of the part and is held stationary. The DTP, on the right side, pushes the part with its bottom probe tip for a distance of about $1100 \mu \mathrm{m}$. From these experiments, we can see that the orientation of the peg is robustly rotated closed to $\pi$ even though uncertainties cause significant mismatches in $x$ and $y$ displacements.

A push of approximately $950 \mu \mathrm{m}$ was used for the robust $x$ translation experiments. The predicted results are within the error margins of the experimental observations.

Combining these three types of robust motions together allows us to execute the planned algorithm described in Section IV-E. Because of the limited controllability of $x$ position of the peg in the current experimental platform and planning algorithm, the initial configuration is set to be $\left(x_{\text {init }}=2060.4 \mu \mathrm{m}, y_{\text {init }}=-9.2 \mu \mathrm{m}, \theta_{\text {init }}=\frac{\pi}{2}\right)$ such that one robust $y$ motion followed by one robust rotation followed by one robust $x$ motion is able to push the peg into the hole. A simulation of the planned motion is shown in Fig. 10 (left). In the experiments, the peg is successfully pushed into the hole three times over three trials. The experimental data from one trial is shown in Fig. 10 (right). The snapshots of the experiment are shown in Fig. 11 with the associated robust controls.

\section{CONCLUSION}

In this paper, we established a framework for motion planning under differential constraints and uncertainties in sensing, control (actuation), and geometric/dynamic parameters. We show how we can characterize robust motion primitives with applications to quasi-static manipulation and assembly 

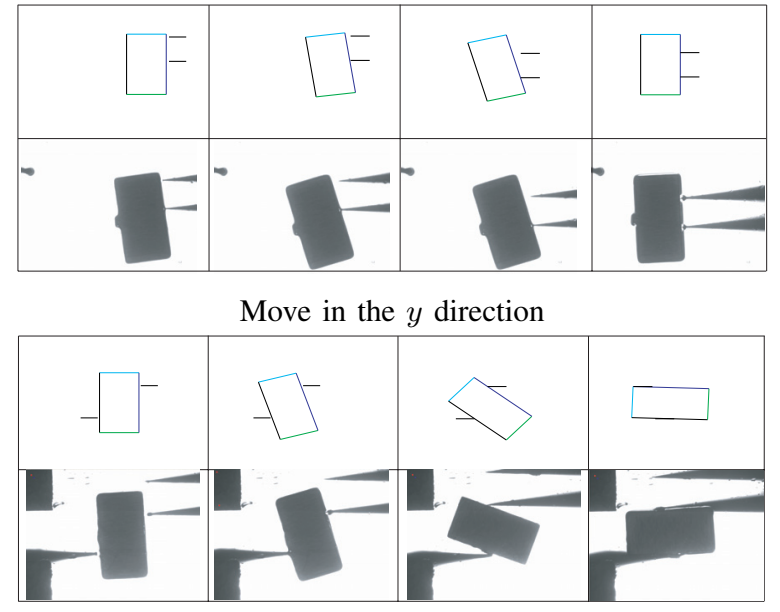

Rotate to the $\theta=\pi$ configuration

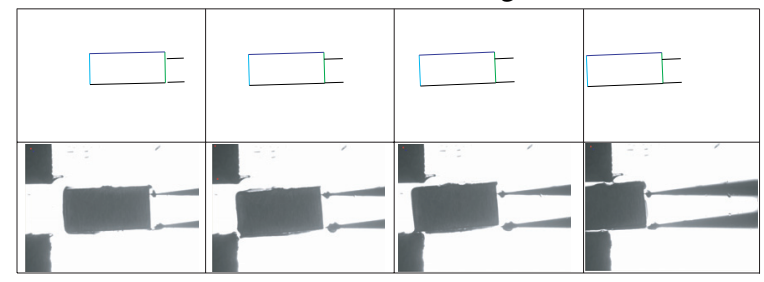

Translate the part in $x$ direction to the goal

Fig. 11. Snapshots illustrating the assembly of the part into the slot.

tasks and propose measures to quantify robustness of motion primitives. Further, we describe an algorithm to automatically synthesize motion plans which sequentially compose robust motion primitives to move parts to goal positions with minimal actuation.

The main contribution in this paper is the quantitative treatment of uncertainty and the incorporation of models of uncertainty into the synthesis of motion primitives and the motion plans. It is clear that this paper is only a starting point and does not address the problems associated with multipoint contact which characterize assembly tasks. Further, we simplified the modeling of the contact friction by considering lubricated surfaces which appear to be well modeled by viscous damping. Nevertheless, the ability to plan and reliably execute the plan for positioning and orienting parts using visual feedback with only a single degree-of-freedom actuator represents a significant accomplishment over previous studies on quasi-static manipulation.

\section{ACKNOWLEDGMENTS}

We gratefully acknowledge the support of NSF grants DMS01-39747, IIS02-22927, and IIS-0413138, and ARO grant W911NF-04-1-0148.

\section{REFERENCES}

[1] S. Akella and M. T. Mason. Posing polygonal objects in the plane by pushing. International Journal of Robotics Research, 17(1):70-88, January 1998 .

[2] J.C. Alexander and J.H. Maddocks. Bounds on the friction-dominated motion of a pushed object. The International Journal of Robotics Research, 12(3):231-248, June 1993.

[3] M. Anitescu and F.A. Potra. Formulating dynamic multi-rigid-body contact problems with friction as solvable linear complementarity problems. Nonlinear Dynamics, 14:231-247, 1997.
[4] Z. Balorda. Reducing uncertainty of objects by robot pushing. IEEE Int. Conf. on Robotics and Automation, pages 1051-1056, 1990.

[5] S. Berard, B. Nguyen, B. Roghani, J. Trinkle, J. Fink, and V. Kumar. Davincicode: A multi-model simulation and analysis tool for multibody systems. In Proceedings of the IEEE International Conference on Robotics and Automation, 2007.

[6] K. Boehringer, R.Fearing, and K. Goldberg. Handbook of Industrial Robotics, 2nd Ed., chapter Microassembly, pages 1045-1066. John Wiley and Sons, 1999.

[7] D.J. Cappelleri, J. Fink, and V. Kumar. Modeling uncertainty for planar meso-scale manipulation and assembly. ASME 2006 International Design Engineering Technical Conference (IDETC), Philadelphia, PA, 2006.

[8] P. Cheng. Sampling-Based Motion Planning with Differential Constraints. PhD thesis, University of Illinois, Urbana, IL, August 2005.

[9] P. Cheng and V. Kumar. Sampling-based falsification and verification of controllers for continuous dynamic systems. In S. Akella, N. Amato, W. Huang, and B. Misha, editors, Workshop on Algorithmic Foundations of Robotics VII, 2006.

[10] H. Choset, K. M. Lynch, S. Hutchinson, G. Kantor, W. Burgard, L. E Kavraki, and S. Thrun. Principles of Robot Motion: Theory, Algorithms, and Implementations. MIT Press, Cambridge, MA, 2005.

[11] M.A. Erdmann and M.T. Mason. An exploration of sensorless manipulation. IEEE Journal of Robotics and Automation, 4(4), August 1998.

[12] R.S. Fearing. Survey of sticking effects for micro parts handling. IEEE/RSJ Int. Conf. on Intelligent Robotics and Sys.(IROS), Pittsburgh, $P A$, 2:212-217, August 5-9 1995.

[13] K.Y. Goldberg. Orientating polygonal parts without sensing. Algorithmica, 10(2/3/4):210-225, August/September/October 1993.

[14] Suresh Goyal, Elliott N. Pinson, and Frank W. Sinden. Simulation of dynamics of interacting rigid bodies including friction i: General problem and contact model. Engineering with Computers, 10:162-174, 1994.

[15] S. M. LaValle. Planning Algorithms. Cambridge University Press, Cambridge, U.K., 2006. Available at http://planning.cs.uiuc.edu/.

[16] K.M. Lynch. The mechanics of fine manipulation by pushing. IEEE Int. Conf. on Robotics and Automation, Nice, France, pages 2269-2276, May 1992.

[17] K.M. Lynch. Estimating the friction parameters of pushed objects. Proc. 1993 IEEE/RSJ Int. Conf., July 1993.

[18] K.M. Lynch and M.T. Mason. Stable pushing: Mechanics, controllability, and planning. International Journal of Robotics Research, 15(6):553-556, December 1996.

[19] M.T. Mason. Mechanics and planning of manipulator pushing operations. International Journal of Robotics Research, 5(3):53-71, 1986.

[20] M. Moll, K. Goldberg, M.A. Erdmann, and R. Fearing. Orienting microscale parts with squeeze and roll primitives. IEEE Int. Conf. on Robotics and Automation, Washington, DC, May 11-15 2002.

[21] J.S. Pang and J.C. Trinkle. Complementarity formulations and existence of solutions of dynamic multi-rigid-body contact problems with coulomb friction. Mathematical Programming, 73:199-226, 1996.

[22] M.A. Peshkin and A.C. Sanderson. The motion of a pushed, sliding object, part1: Sliding friction. Technical Report CMU-RI-TR-85-18, Robotics Institute, Carnegie Mellon University, Pittsburgh, PA, September 1985 .

[23] D.T. Pham, K.C. Cheung, and S.H. Yeo. Initial motion of a rectangular object being pushed or pulled. IEEE Int. Conf. on Robotics and Automation, 1046-1050, 1990.

[24] M. Salganicoff, G. Metta, A. Oddera, and G. Sandini. A vision-based learning method for pushing manipulation. AAAI Fall Symp. on Machine Learning in Computer Vision., 1993b.

[25] P. Song, J.S. Pang, and V. Kumar. A semi-implicit time-stepping model for frictional compliant contact problems. International Journal for Numerical Methods in Engineering, Accepted for publication 2004.

[26] D. E. Stewart and J.C. Trinkle. An implicit time-stepping scheme for rigid body dynamics with inelastic collisions and coulomb friction. International J. Numer. Methods Engineering, 39(15):281-287, 1996.

[27] J.C. Trinkle, S. Berard, and J.S. Pang. A time-stepping scheme for quasistatic multibody systems. International Symposium of Assembly and Task Planning, July 2005.

[28] T. Yoshikawa and M. Kurisu. Identification of the center of friction from pushing an object by a mobile robot. IEEE/RSJ International Workshop on Intelligent Robots and Systems IROS, November 1991. 